\title{
Discussion on growth, emission and piezoelectric properties of zinc guanidinium phosphate single crystal: a potential candidate for transducer and LED applications
}

\author{
S NANDHINI and P MURUGAKOOTHAN* \\ MRDL, PG and Research Department of Physics, Pachaiyappa's College, Chennai 600 030, India \\ *Author for correspondence (murugakoothan03@yahoo.co.in)
}

MS received 22 October 2018; accepted 8 March 2019

\begin{abstract}
Zinc guanidinium phosphate, a semi-organic single crystal, was grown successfully by a slow evaporation solution growth technique. The cell parameters of the grown crystal were confirmed using a powder X-ray diffraction study. The electrical property of the sample was analysed using dielectric and piezoelectric studies. The dielectric constant, dielectric loss and alternating-current conductivity were calculated for various frequencies and temperatures. The solid state parameters such as plasma energy, Penn gap energy, Fermi energy and electronic polarizability were calculated. The piezoelectric property of the title crystal was ascertained by determining its piezoelectric charge coefficient $\left(d_{33}\right)$. The optical transmittance and energy gap of the grown crystal were examined using ultraviolet-visible-near-infrared spectral analysis. The title molecule was optimized and the frontier molecular orbital was performed using B3LYP/6-31 + G(2d,2p). The emission property of the titular compound was analysed using a photoluminescence study. The dominant colour emission of the grown crystal was found using CIE colour chromaticity coordinates. The nonlinear property of the titular compound was confirmed using a Kurtz-Perry powder technique. The above results illustrate that the title crystal is a potential candidate for light emitting and nonlinear applications. This work explains the dominant colour emitting behaviour and electrical properties such as dielectric and piezoelectric properties of the title crystal, which are reported for the first time.
\end{abstract}

Keywords. Semi-organic crystal; Penn gap energy; piezoelectric; photoluminescence; HOMO-LUMO.

\section{Introduction}

The art of growing nonlinear optical (NLO) crystals becomes a fascinating field for the researchers due to their significant role in emerging photonic and optoelectronic technologies [1]. NLO crystals can be broadly classified into organic, inorganic and semi-organic crystals. Each type of crystals has its own advantages and disadvantages. In this, semi-organic compounds are of high interest because of their high polarizability as compared with inorganic compounds and good thermomechanical behaviour as compared with organic compounds. This makes the researchers to concentrate on developing some optically good semi-organic single crystals.

Guanidine compounds are potentially interesting candidates for the NLO applications, due to the presence of six donor sites for hydrogen bonding interactions and delocalized electron systems [2]. In recent years, many guanidinebased NLO crystals such as guanidinium 4-nitrobenzoate [3], guanidinium 4-aminobenzoate [4], zinc guanidinium sulphate [5] and guanidinium L-monohydrogen tartrate [6] were reported. The structural, spectral, NMR, thermal and hardness studies of zinc guanidinium phosphate (ZGuP) were already reported [7]. In the present work, a detailed discussion on dielectric, piezoelectric, optical, highest occupied molecular orbital-lowest unoccupied molecular orbital
(HOMO-LUMO) analysis and emission properties of a semi-organic single crystal, ZGuP is reported.

\section{Synthesis}

The title compound was synthesized using guanidine carbonate, zinc sulphate and orthophosphoric acid in the ratio of $1: 1: 3$ and dissolved in deionized water. The solution was stirred well using a magnetic stirrer in order to attain homogeneity. The solution was then filtered and kept undisturbed. By employing a slow evaporation solution growth technique and in the growth period of 32 days, an optically good quality ZGuP crystal with a size of $14 \times 6 \times 3 \mathrm{~mm}^{3}$ was harvested and is shown in figure 1 .

\section{Results and discussion}

\subsection{PXRD study}

The cell parameters of the title compound were determined using a powder X-ray diffraction (PXRD) study. The recorded PXRD spectrum of $\mathrm{ZGuP}$ is shown in figure 2. 


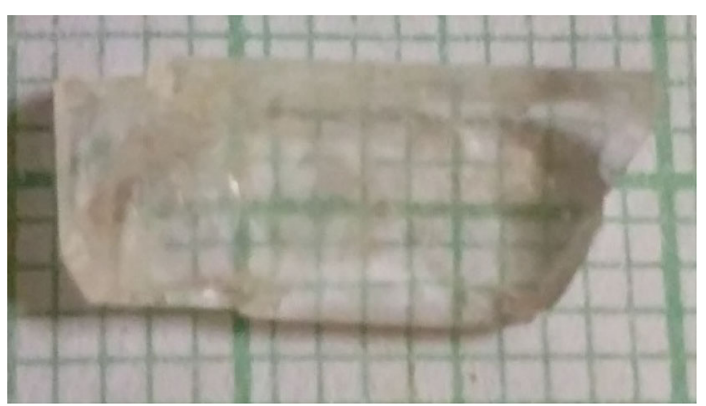

Figure 1. As grown $\mathrm{ZGuP}$ single crystal.

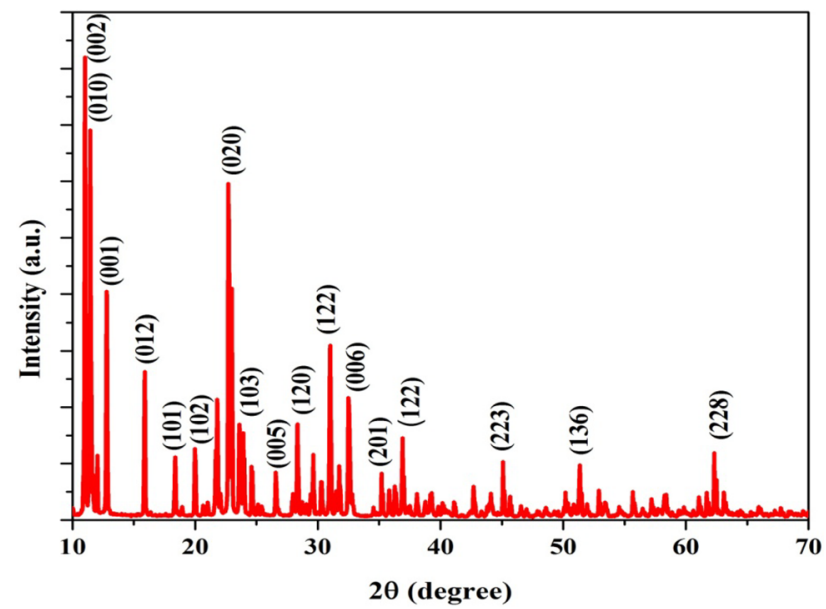

Figure 2. PXRD spectrum of the ZGuP crystalline sample.

In the obtained spectrum, we could see the sharp diffraction peaks and low full-width at half maximum value, which clearly shows a better crystalline nature of the grown crystal. The title crystal belongs to the monoclinic crystal system with the cell parameters of $a=5.132 \AA, b=$ $7.841 \AA, c=16.510 \AA, \alpha=\gamma=90^{\circ}$ and $\beta=90.119^{\circ}$. It is a non-centrosymmetric crystal with the space group $P_{21}$. The obtained cell parameters are in good agreement with the reported value [8].

\subsection{Dielectric study}

The electrical property of the grown crystal was determined from dielectric studies. A well-polished, optically defect free crystal was employed for analysis. The dielectric constant and dielectric loss of the ZGuP crystal was recorded at various temperatures with variation in frequencies. The resultant spectra are shown in figure $3 a$ and $b$. From the spectra, we could see that the dielectric constant gradually decreased as the frequency increased. The high dielectric constant at a lower frequency is due to the presence of all kinds of polarizations, as the frequency increases, the contribution of space charge polarization decreases. The lack of space charge polarization attributes to the low dielectric constant at a higher frequency. The dielectric loss graph depicts that it strongly depends on the frequency. The crystal shows a low dielectric loss value at a higher frequency. This behaviour reveals that the grown crystal possesses lesser defects, which is a necessary factor for the fabrication of NLO devices.

The alternating-current (ac) conductivity $\left(\sigma_{\mathrm{ac}}\right)$ of the grown crystal was calculated using the following expression (1):

$$
\sigma_{\mathrm{ac}}=2 \pi f \varepsilon^{\prime} \varepsilon_{0} \tan \delta
$$

where $f$ is the applied frequency, $\varepsilon^{\prime}$ is the relative dielectric permittivity and $\tan \delta$ is the dielectric loss factor. The variation of ac conductivity with respect to the applied frequency is shown in figure $3 \mathrm{c}$.

\subsection{Solid state parameters}

Solid state parameters such as plasma energy, Fermi energy, Penn gap energy and electronic polarizability were calculated from the molecular weight $(M)$, number of valence electrons $(Z)$, density $(\rho)$ and dielectric constant $(\varepsilon)$ of the title crystal. These values help in analysing the polarizability and electrical properties of the material [9].

The molecular density $(\rho)$ of the title crystal was calculated using the equation (2):

$$
\rho=M Z / N V_{\mathrm{A}},
$$

where $M=478.78$, volume of the unit cell, $V=664.36$, $Z=2$ and the $\rho$ value was found to be $2.39 \mathrm{~g} \mathrm{~cm}^{-3}$. The valence electron plasma energy $\left(\hbar \omega_{\mathrm{p}}\right)$ was calculated using the relation (3):

$$
\hbar \omega_{\mathrm{p}}=28.8(Z \rho / M)^{1 / 2}
$$

Using the calculated plasma energy, the Penn gap energy $\left(E_{\mathrm{p}}\right)$ and the Fermi energy $\left(E_{\mathrm{F}}\right)$ were determined using the expressions (4) and (5) respectively,

$$
\begin{aligned}
& E_{\mathrm{p}}=\hbar \omega_{\mathrm{p}}\left(\varepsilon_{\mathrm{r}}-1\right)^{1 / 2}, \\
& E_{\mathrm{F}}=0.2948\left(\hbar \omega_{\mathrm{p}}\right)^{4 / 3} .
\end{aligned}
$$

Using the calculated Penn gap energy and Fermi energy, the electronic polarizability, $\alpha$ of the title compound was determined using the following relation (6):

$$
\alpha=\left[\left(\hbar \omega_{\mathrm{p}}\right)^{2} S_{0} /\left(\hbar \omega_{\mathrm{p}}\right)^{2} S_{0}+3 E_{\mathrm{p}}^{2}\right]
$$

where $S_{0}$ is a constant and its value was calculated using the equation (7):

$$
S_{0}=1-\left[E_{\mathrm{p}} / 4 E_{\mathrm{p}}\right]+1 / 3\left[E_{\mathrm{p}} / 4 E_{\mathrm{F}}\right]^{2} .
$$



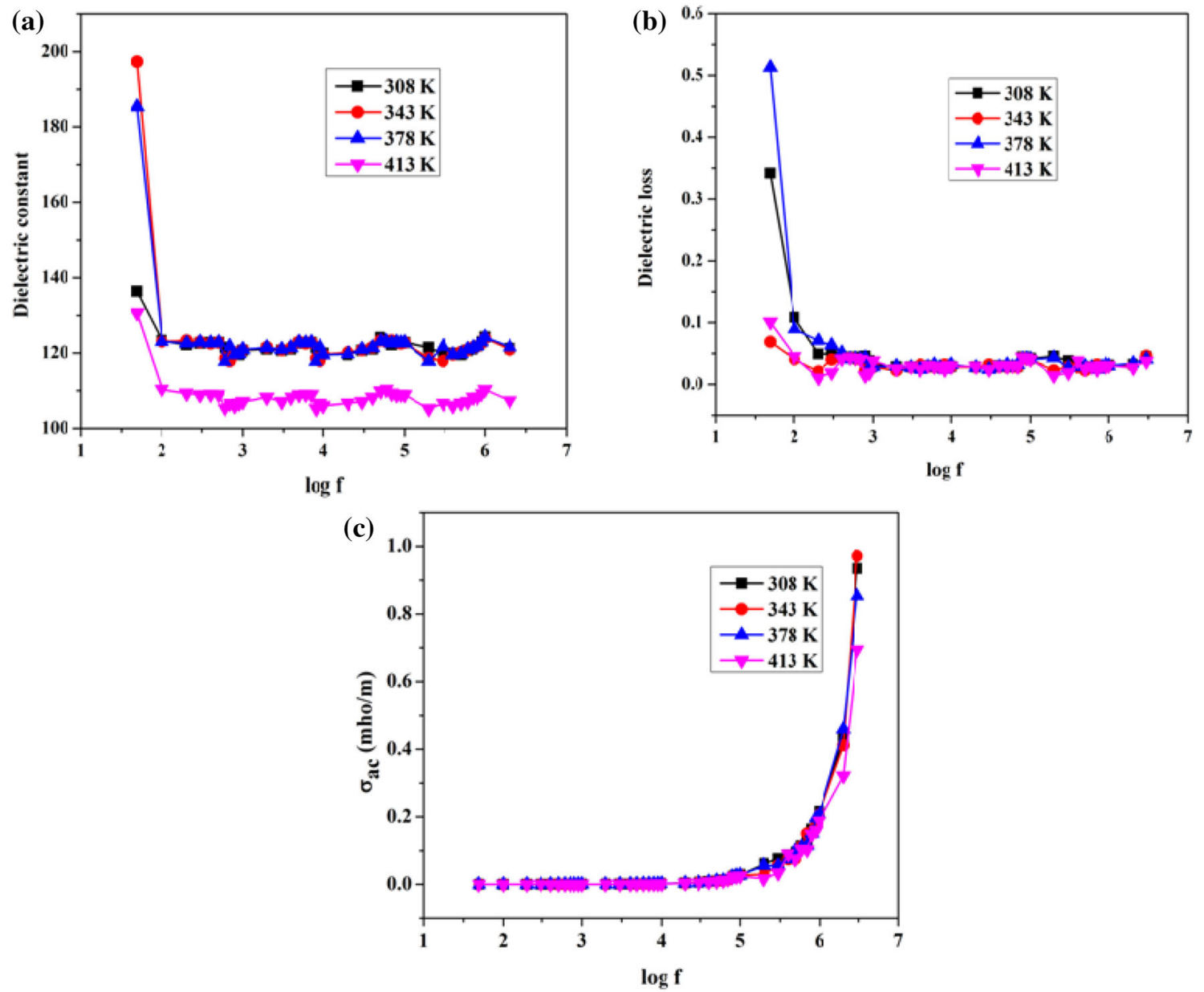

Figure 3. (a) Variation of dielectric constant with frequency of ZGuP, (b) variation of dielectric loss with frequency of ZGuP and (c) ac conductivity of the ZGuP single crystal.

Table 1. The comparison of the electrical properties for the ZGuP single crystal with the KDP crystal.

\begin{tabular}{lcc}
\hline Parameters & Values of ZGuP crystal & Values of KDP crystal [16] \\
\hline Plasma energy $\hbar \omega_{\mathrm{p}}(\mathrm{eV})$ & 18.086 & 17.33 \\
Penn gap energy $E_{\mathrm{p}}(\mathrm{eV})$ & 1.651 & 2.39 \\
Fermi energy $E_{\mathrm{F}}(\mathrm{eV})$ & 13.861 & 12.02 \\
Electronic polarizability $(\alpha)$ using Penn analysis $\left(\mathrm{cm}^{3}\right)$ & $7.206 \times 10^{-23}$ & $2.14 \times 10^{-23}$ \\
Electronic polarizability $(\alpha)$ using Clausius-Mossotti equation $\left(\mathrm{cm}^{3}\right)$ & $7.750 \times 10^{-23}$ & $2.18 \times 10^{-23}$ \\
\hline
\end{tabular}

The value of $\alpha$ was also calculated from the ClausiusMossotti relation (8):

$$
\alpha=\left(3 M / 4 \pi N_{\mathrm{a}}\right)\left\{\left(\varepsilon_{\mathrm{r}}-1\right) /\left(\varepsilon_{\mathrm{r}}+2\right)\right\},
$$

where $\varepsilon_{\mathrm{r}}$ is the dielectric constant $\left(\varepsilon_{\mathrm{r}}=121\right.$ at $\left.4 \mathrm{MHz}\right)$ of the title compound and $N_{\mathrm{a}}$ is the Avogadro number.

The calculated solid state parameters are listed in table 1 and are compared with the potassium dihydrogen phosphate (KDP) crystal. The obtained values are higher than those of the reported KDP crystal and show that ZGuP are comparably better than the KDP crystal.

\subsection{Piezoelectric study}

The ability of a material to produce an electrical charge on applying mechanical stress is termed as piezoelectric effect. Crystal symmetry plays a significant role in determining the piezoelectric effect. In general, larger is the polarizability greater will be the piezoelectric coefficient of the material. 
In the present work, the piezoelectric property was confirmed by determining the piezoelectric charge coefficient $\left(d_{33}\right)$. The piezoelectric coefficient of the title crystal was found to be $4 \mathrm{pC} \mathrm{N}^{-1}$. The obtained value is considerably higher than some of the reported compounds and is listed in table 2. This shows that the ZGuP material finds its applications in transducer devices, sensing entity on electronic micro-chips and electric voltage sources [10].

\subsection{UV-Vis-NIR spectral analysis}

The linear optical property of the grown crystal was analysed using a ultraviolet-visible-near-infrared (UV-Vis-NIR)

Table 2. Comparison of the piezoelectric property with other reported materials.

\begin{tabular}{lc}
\hline Compound & $\begin{array}{r}\text { Piezoelectric coefficient } \\
\left(d_{33}\right) \mathrm{pC} \mathrm{N}^{-1}\end{array}$ \\
\hline ZGuP & 4 \\
$\begin{array}{l}\text { Sodium } p \text {-nitrophenolate } \\
\text { dehydrate }\end{array}$ & 2.24 \\
Triglycine sulphate & 1 \\
\hline
\end{tabular}

spectral study. A well-polished optically transparent crystal was used for the study. The obtained transmittance spectrum is shown in figure $4 \mathrm{a}$. The ZGuP crystal shows $52 \%$ transparency and a lower cut-off wavelength of $227 \mathrm{~nm}$ which is due to the transition of electrons between $\pi$ and $\pi *$ transition states. The enhanced optical transmittance reveals that the grown crystal possesses less structural defects, which in turn may reduce scattering in its planes. The above result shows that the ZGuP crystal is a potential candidate for optical device applications.

3.5a Determination of optical constants: The absorption coefficient $(\alpha)$ is an essential factor to find the energy gap of a material. It relates the transmittance $(T)$ and thickness of the material $(t)$ by the following relation (9):

$$
\alpha=2.3026 / t \log (1 / T) .
$$

The band gap energy of the crystal was determined using Tauc's plot. The following expression (10) relates the absorption coefficient $(\alpha)$ and photon energy $(E)$, which was used to calculate the energy gap of the material,

$$
(\alpha h v)^{2}=A\left(E_{\mathrm{g}}-h v\right) .
$$
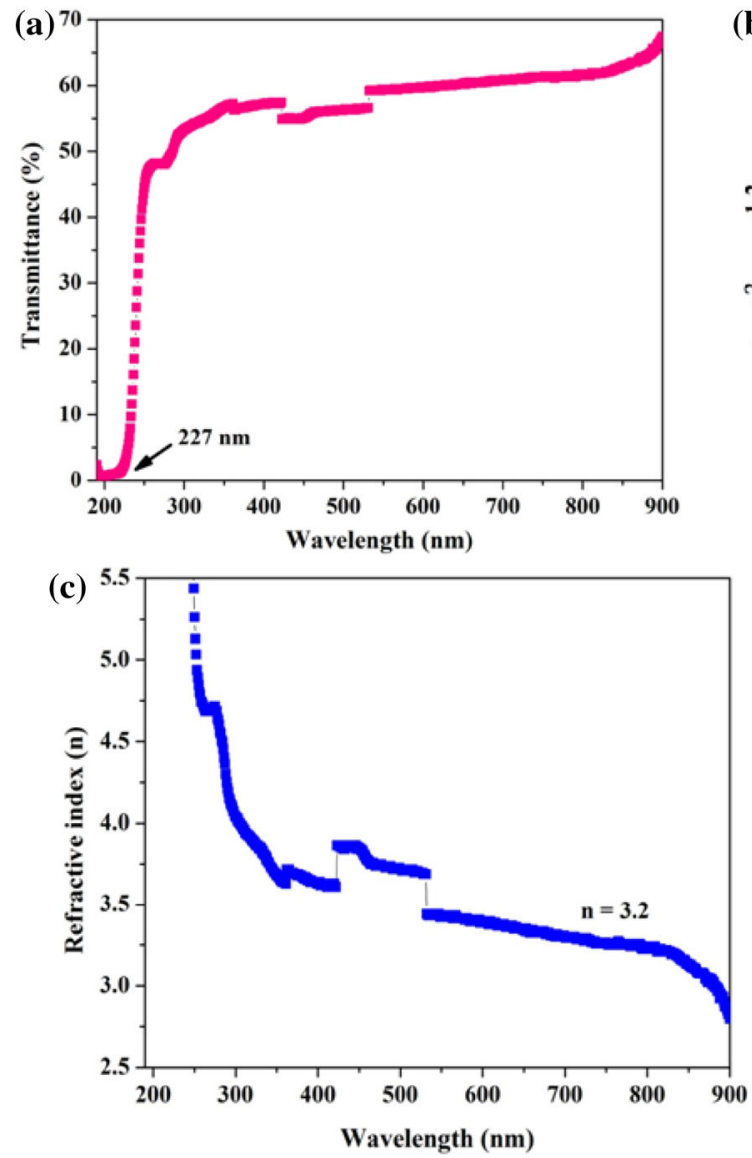
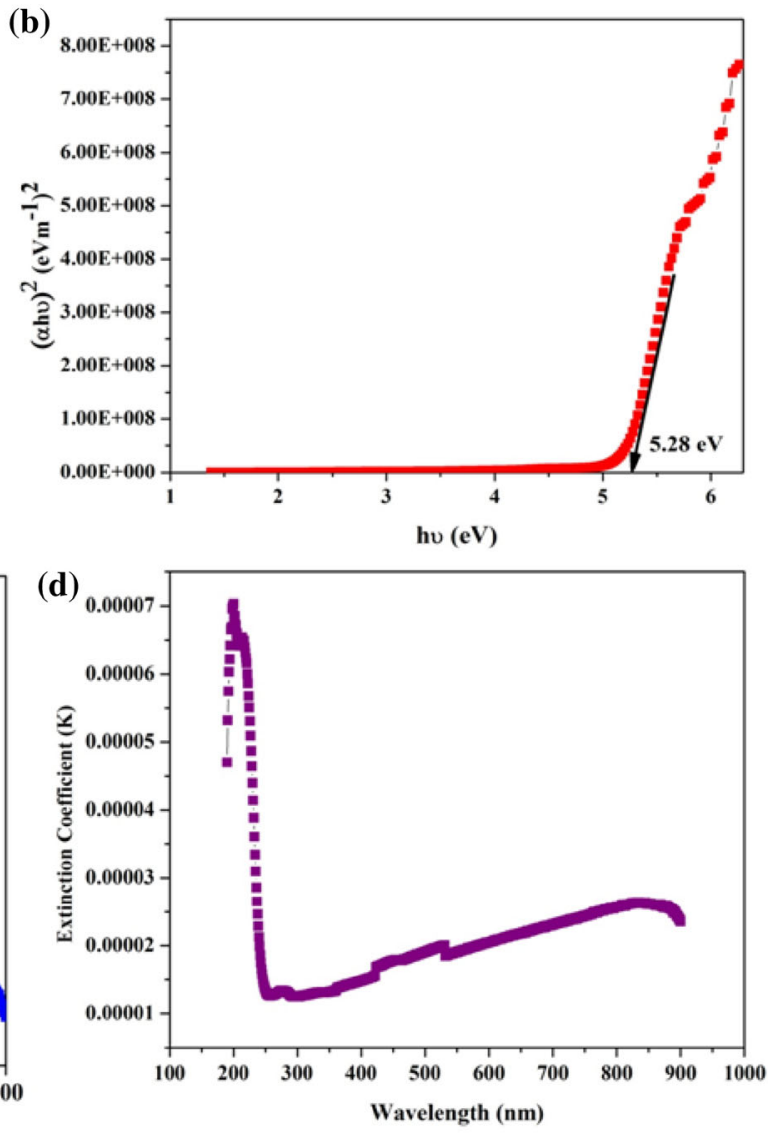

Figure 4. (a) UV-Vis-NIR transmittance spectrum, (b) Tauc's plot, (c) refractive index and (d) extinction coefficient of the ZGuP crystal. 


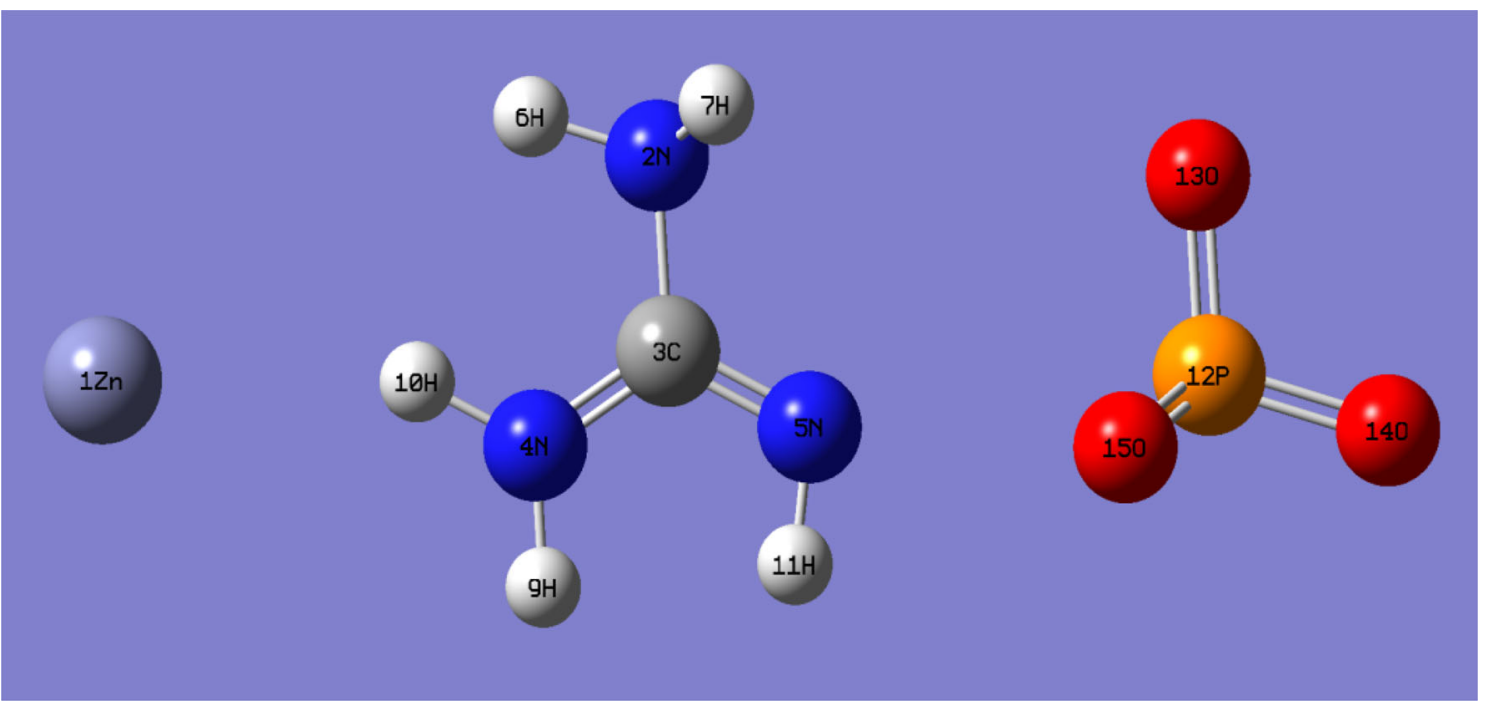

Figure 5. Optimized molecular structure of ZGuP.

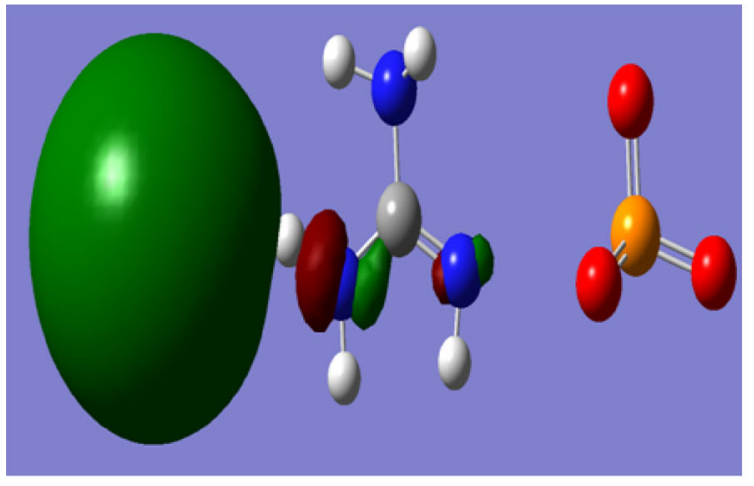

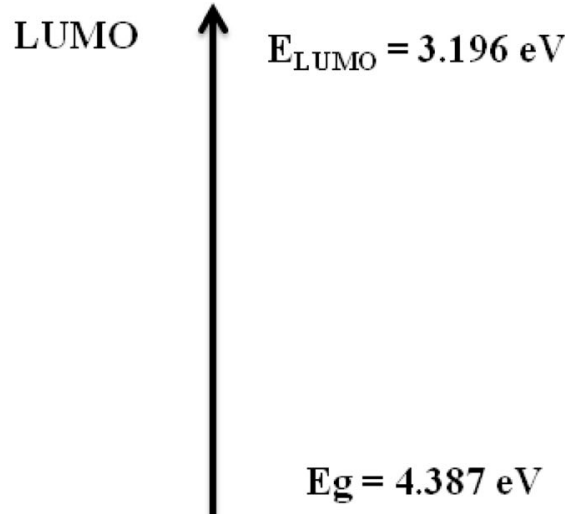

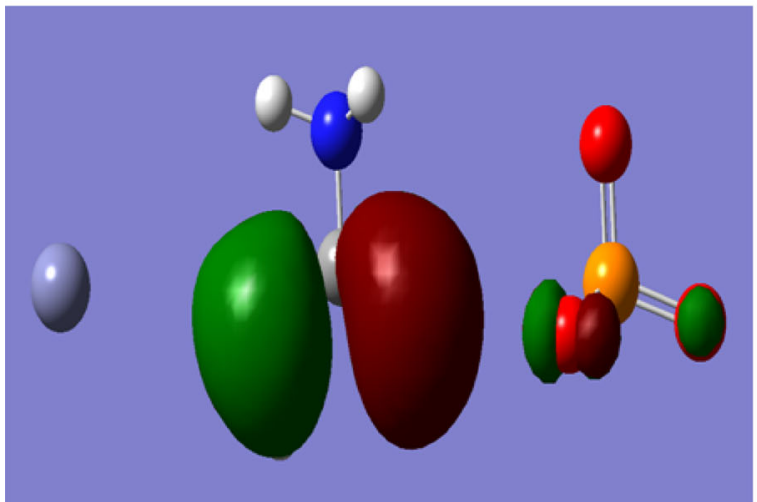

HOMO

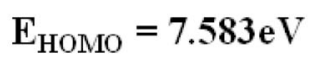

Figure 6. Frontier molecular orbitals of ZGuP.

The Tauc's plot of the title crystal is shown in figure $4 \mathrm{~b}$. The energy gap was determined by extrapolating the linear part of the graph to energy axis i.e., $(\alpha h v)^{2}=0$. The band gap energy of the ZGuP crystal was calculated as $5.28 \mathrm{eV}$. This shows that the material is having wide band gap energy. 
The extinction coefficient $(K)$, reflectance $(R)$ and refractive index $(n)$ were determined using the following expressions (11), (12) and (13) respectively:

$$
\begin{aligned}
K= & \lambda \alpha / 4 \pi, \\
R= & \{\exp (-\alpha t)+[\exp (-\alpha t) T-\exp (-3 \alpha t) T \\
& \left.\left.+\exp (-\alpha t) T^{2}\right]^{1 / 2}\right\} /[\exp (-\alpha t)+\exp (-2 \alpha t)], \\
n= & {\left[(R+1)+\left(3 R^{2}+10 R-3\right)^{1 / 2}\right] /[2(R-1)] . }
\end{aligned}
$$

Figure $4 \mathrm{c}$ and $\mathrm{d}$ shows the variation of the refractive index and extinction coefficient of the $\mathrm{ZGuP}$ crystal with respect to the wavelength. Both the parameters decrease gradually as the wavelength increases. The refractive index of the grown crystal was found as 3.2.

\subsection{Frontier molecular orbital analysis}

The computational calculations for the title compound were performed using Gaussian 09 software [11] and viewed in the Gauss view program [12]. The structure of ZGuP molecule was optimized using the B3LYP/6-31 + (2d,2p) basis set. The optimized geometry of the title molecule is shown in figure 5. Using the optimized geometry, the frontier molecular orbital calculation was carried out and the corresponding HOMO-LUMO orbital was determined and is shown in figure 6. The energy gap value was determined from HOMO-LUMO analysis. The energy gap of the title molecule was found as $4.387 \mathrm{eV}$. The slight discrepancy between the theoretical and experimental energy gap is due to the fact that the theoretical calculations were performed for the individual molecule at the gas phase.

\subsection{PL study}

The photoluminescence (PL) spectrum of the grown crystal was recorded at room temperature. The excitation wavelength was fixed at $250 \mathrm{~nm}$ and the excitation spectrum is shown in figure 7 . The related emission peak was observed at $409 \mathrm{~nm}$ and the corresponding emission spectrum is shown in figure 8 . The emission peak shows that the ZGuP crystal is an excellent light emitting material. It is understood from the high intensity of PL emission that the low concentration of defects in the grown crystal which make the grown crystal as a potential candidate for optical device applications [13]. CIE colour chromaticity coordinates explain the dominant colour emission of the sample and is shown in figure 9. The values of the colour chromaticity coordinates $(x, y)$ are found to be $(0.1677,0.1354)$. The obtained result shows that the grown crystal can be used as a desired candidate for solid state lightning applications [14].

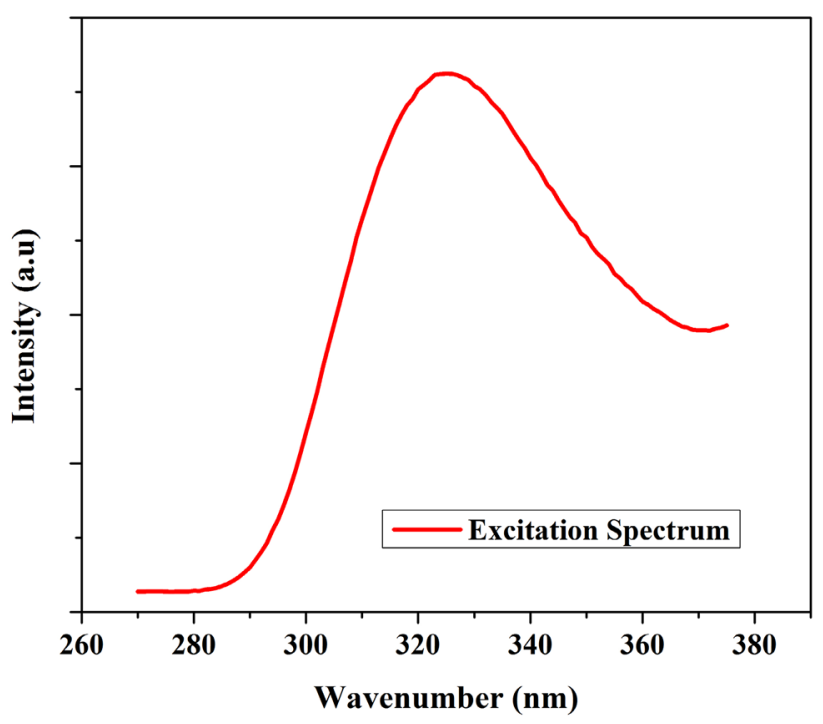

Figure 7. PL excitation spectrum of the ZGuP crystal.

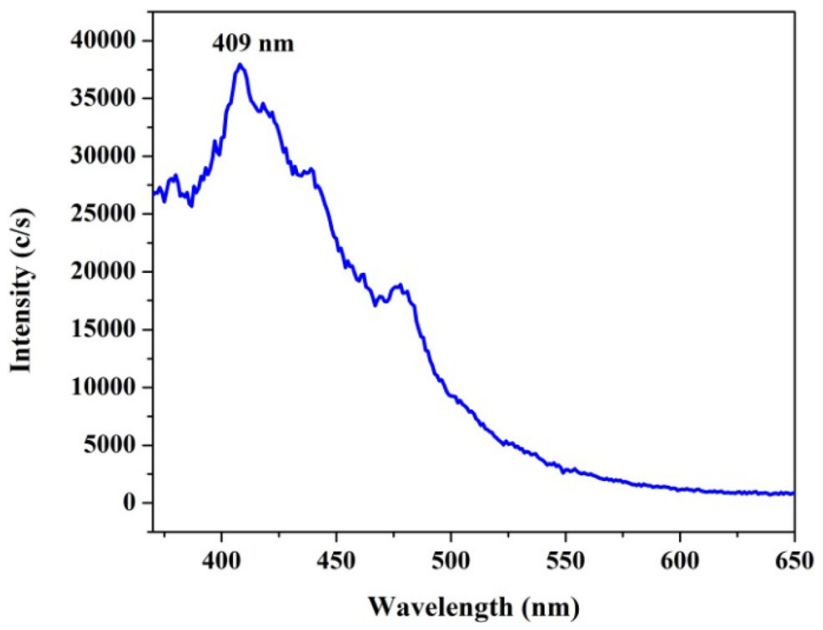

Figure 8. PL emission spectrum of the ZGuP crystal.

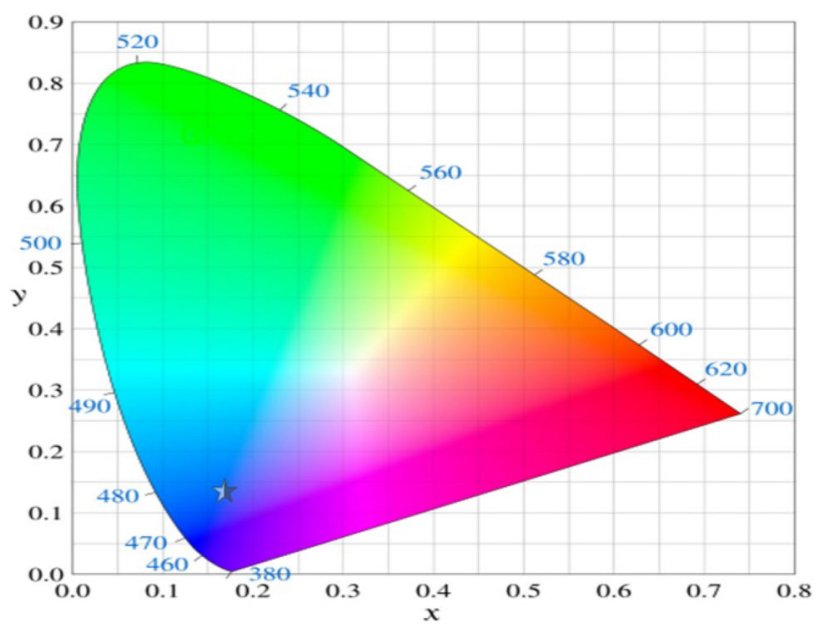

Figure 9. CIE colour chromaticity diagram of the ZGuP crystal. 


\subsection{Second harmonic generation property}

The frequency conversion property of the titular compound was examined using the Kurtz-Perry powder technique [15]. The laser source with a wavelength of $1064 \mathrm{~nm}$, a pulse width of $8 \mathrm{~ns}$ and a repetition rate of $10 \mathrm{~Hz}$ was illuminated to the sample. The input energy of $1 \mathrm{mV}$ was supplied to the sample, which in turn resulted in an output energy of $42 \mathrm{mV}$. The output energy of the title compound was compared with the standard KDP crystal, which showed $24 \mathrm{mV}$ as the output energy. The output of the ZGuP crystal was found to be 1.75 times compared to that of the KDP crystal. The obtained result reveals that the title crystal could be a suitable candidate for nonlinear applications.

\section{Conclusions}

A potential NLO single crystal, ZGuP was grown by a slow evaporation solution growth technique. The ZGuP crystal belongs to the monoclinic crystal system with the non-centrosymmetric space group $P_{21}$. The low dielectric constant and low dielectric loss show that the crystal possesses lesser defects and can be employed for NLO devices. The piezoelectric coefficient $\left(d_{33}\right)$ value $4 \mathrm{pC} \mathrm{N}^{-1}$ of the title crystal shows that it could be employed for transducer applications. The optical property shows that the crystal has good transparency in the entire visible region and a lower cut-off wavelength of $227 \mathrm{~nm}$. The molecular structure of the title compound was optimized and the HOMO-LUMO analysis showed an energy gap of $4.387 \mathrm{eV}$. The PL study shows that the significant emission of the title compound occurs at $409 \mathrm{~nm}$. The CIE colour chromaticity diagram shows that the title crystal emits blue colour dominantly and hence, it could be used as a suitable candidate for optical applications. The titular crystal shows SHG property 1.75 times the KDP crystal. The solid state parameters show a better result on compared with the KDP crystal.

\section{References}

[1] Mohanraj K, Balasubramanian D and Jhansi N 2017 Opt. Laser Technol. 96318

[2] Petrosoyan A M, Sukiyasan R P, Karpetyan H A, Tenzya S S and Feigelson R S 2000 J. Cryst. Growth 213103

[3] Kleb D C, Schurmann M, Preut H and Bleckmann P 1998 Z. Kristallogr. New. Cryst. Struct. 213581

[4] Pereira Silva P S, Ramos Silva M, Paixao J A and Matos Beja A 2010 Acta Cryst. E66 0524

[5] Sivashankar V, Siddheswaran R, Bharthasarathi T and Murugakoothan P 2009 J. Cryst. Growth 3112709

[6] Zyss J, Pecaut J, Levy J P and Masse R 1993 Acta Cryst. B49 334

[7] Suvitha A and Murugakoothan P 2012 Spectrochim. Acta A 86 266

[8] Harrison W T A 1997 Chem. Mater. 91837

[9] Sivasubramani V, Mohankumar V, Senthil Pandian M and Ramasamy P 2017 CrystEngComm 195662

[10] Bune A V, Zhu C and Ducharme S 1999 J. Appl. Phys. 857869

[11] Gaussian 09, 2009 Revision A.2, Frisch M J, Trucks G W, Schlegel H B, Scuseria G E, Robb M A, Cheeseman J R et al Fox, Gaussian Inc, Wallingford CT

[12] Gaussview 2006 Version 4.1.2., Gaussian Inc, Wallingford CT

[13] Shanmugam G and Bragadeshwaran S 2012 Spectrochim. Acta A 95177

[14] Vijayakumar R and Marimuthu K 2015 J. Mol. Struct. 1092 166

[15] Kurtz K and Perry T T 1968 J. Appl. Phys. 363798

[16] Robert R, Justin Raj C, Krishnan S and Jerome Das S 2010 Physica B $\mathbf{4 0 5} 20$ 